\title{
EVALUACIÓN DE LA INFORMACIÓN SOBRE OCUPACIÓN DEL SUELO EN ESPAÑA PARA ESTIMAR LA SUPERFICIE FORESTAL EN GALICIA
}

\author{
Eduardo Daniel García Martínez \\ María Luisa Chas-Amil \\ Departamento de Economía Cuantitativa. Universidade de Santiago de Compostela \\ edgm_73@yahoo.es, marisa.chas@usc.es \\ Julia Touza \\ Environment Department. University of York, UK \\ julia.touza@york.ac.uk
}

La identificación y mapeo de la superficie forestal son de extrema importancia para la investigación en el área ambiental y el desarrollo de políticas de gestión del territorio y de los recursos naturales. El estudio de la biomasa, captura y secuestro de carbono, impactos ambientales, evaluaciones sobre la conservación de los bosques y su biodiversidad depende de un conocimiento detallado de la superficie forestal. Por tanto, las políticas relativas a la planificación territorial, tales como las repoblaciones forestales, prevención y mitigación de incendios forestales, reducción del abandono rural, incentivos a la industria forestal, o conservación de hábitats amenazados, entre otras, requieren de esta información sobre los recursos forestales, su localización y características.

En este artículo nos centramos en la evaluación de la armonización en la cuantificación del espacio forestal a través de las fuentes disponibles, que en los últimos tiempos vienen utilizando técnicas cartográficas y de fotointerpretación. En España la descripción pormenorizada de las características de los montes se viene recogiendo en los Inventarios Forestales Nacionales que incluyen cartografía en formato digital desde la publicación del Segundo Inventario Forestal Nacional (1986-1996). Recientemente ya está disponible el Cuarto Inventario Forestal Nacional (IFN4) para algunas Comunidades Autónomas (CCAA) españolas. El IFN4 mejora sustancialmente la calidad de la información del Tercer Inventario Forestal (IFN3) (1995-1998) en relación, especialmente, a la escala utilizada (1:25.000) y a las unidades mínimas cartografiables. Además, se ha mejorado la estructura de base de datos, que es más manejable y con una capacidad de explotación mucho mayor.

A nivel europeo, se dispone de información relativa a la discriminación de usos del suelo, incluido el forestal, a través de las coberturas del proyecto CORINE Land Cover (CLC) 
para los años 1990 (CLC90), 2000 (CLC00) y 2006 (CLC06). Sin embargo, a pesar del gran avance que ha supuesto tener una fuente de información comparable entre países, la falta de resolución del CLC deja patente la necesidad de disponer de mayor información a nivel estatal y regional. Esto es debido a que el CLC tiene una escala de referencia de 1:100.000, lo que no ofrece una resolución suficiente para estudios que requieran de cierto nivel de detalle, no permitiendo una medición precisa de los diferentes usos de suelo. Además, tanto la información de referencia del CLC como la escala de trabajo y las unidades mínimas cartografiables, 25 hectáreas (ha), condicionan enormemente la precisión, lo que repercute en los resultados obtenidos. De ahí que hayan surgido proyectos a nivel de las CCAA españolas (ej. Mapa de Coberturas e Usos do Solo (USO_25) para el caso de Galicia), o a nivel estatal como el Sistema de Ocupación del Suelo de España (SIOSE). El SIOSE, puesto en marcha por el Instituto Geográfico Nacional (IGN), nació con el objetivo de generar una base de datos de ocupación y uso del suelo para toda España integrando la información disponible por las CCAA y la Administración General del Estado.

El SIOSE tiene una primera versión con datos de ocupación con referencia el año 2005 (SIOSE05) y en la actualidad está disponible la tercera actualización con fecha de referencia el año 2011. Los atributos principales a destacar del SIOSE son su mayor resolución y precisión en comparación con otras opciones cartográficas de coberturas del suelo, tales como el CLC ya comentada en el párrafo anterior. La escala de referencia del SIOSE es 1:25.000, constituyéndose, por tanto, en la primera capa continua para todo el estado español a ese nivel de detalle. Utiliza un modelo de datos que permite integrar las nomenclaturas ya existentes en otras fuentes, como el CLC o el IFN4. Pero con la particularidad de que el territorio está dividido en teselas con una o varias coberturas asociadas, con la única restricción de representar una superficie mínima de entre 0,5 y 2 ha en función de los elementos a cartografiar. Estas son grandes ventajas, si se comparan estos atributos del SIOSE con las características gráficas de otras fuentes cartográficas. Sin embargo, el SIOSE05 tampoco está exento de problemas. La explotación de la información de la base de datos resulta laboriosa. Debido a la complejidad del modelo de datos, no es posible la representación gráfica sobre el terreno de las teselas de tipo mixto, es decir, aquellas caracterizadas por agrupar coberturas del suelo múltiples. Esto imposibilita, la obtención de la parte gráfica de los diferentes usos del suelo ya que estos sólo se reflejan a través de porcentajes de ocupación en la base de datos. Por este motivo sólo se puede extraer de forma alfanumérica la información relativa a cada cobertura del suelo pero no la parte gráfica. Esta carencia influye en la capacidad de disponer de la delimitación de las teselas de cualquier cobertura de uso (ej. forestal, agrícola, urbano) y poder realizar las tareas de geoprocesamiento que con otras fuentes cartográficas si es posible.

El objetivo de este trabajo es la evaluación del SIOSE05 como fuente cartográfica de referencia para la planificación del territorio y, en concreto, de la superficie forestal. Teniendo en cuenta la principal limitación ya detallada de esta fuente, este trabajo desarrolla una actualización de la información del SIOSE05, a través de la utilización de ortofotos del Plan Nacional de Ortofotografía Aérea (PNOA) referidas en su mayoría a los años 2007 y 2008 . Esto nos permite delimitar, de una forma precisa, el espacio forestal, generando una actualización del SIOSE05, que denotaremos SIOSE07-08. Este estudio ha comparado estimaciones de la 
superficie forestal en Galicia a partir de distintas fuentes cartográficas (SIOSE05 y su versión actualizada, SIOSE07-08, USO_25, CLC06 e IFN4). Este tipo de comparaciones permite evaluar la armonización y fiabilidad existente de la información sobre el área forestal. Además, mejora la comprensión de las diferencias y similitudes ofrecidas por las diferentes fuentes lo que permite a los usuarios (ej. gestores del territorio o investigadores) una elección informada de la cartografía que mejor se adapta a sus necesidades.

Como estudio de caso para la evaluación de la superficie forestal, tomamos la Comunidad Autónoma de Galicia por la repercusión que el sector forestal tiene en esta comunidad. En términos de cortas de madera, Galicia genera anualmente casi 7 millones de $\mathrm{m}^{3}, 57 \%$ del total español. Además, el espacio forestal arbolado de Galicia ha observado un progresivo crecimiento en los últimos años debido, en parte, a unas condiciones climáticas favorables y a unas circunstancias socioeconómicas que propician el progresivo abandono del medio rural. Este abandono repercute directamente en una pérdida de la superficie agraria útil y, por consiguiente, en una colonización de vegetación espontánea seguida de formaciones herbáceas y de matorral que, en ausencia de perturbaciones externas, pueden culminar en masas arbóreas. Se analizan aquí también las diferencias espaciales en la superficie forestal de esta Comunidad Autónoma, lo que permite evaluar las realidades y comportamientos diferenciados en este aspecto de las diferentes provincias y comarcas gallegas.

El SIOSE07-08 permite obtener gráficamente un nivel de detalle superior al del resto de las fuentes cartográficas analizadas por dos motivos: (a) la escala de revisión ha variado entre 1:5.000 y 1:3.000 y la de digitalización entre 1:2.000 y 1:1.000; y (b) esta capa no presenta unidades mínimas cartografiables salvo las derivadas de la escala de revisión de las ortofotos utilizadas. Pero además, la medición del espacio forestal proporciona una imagen reciente de la extensión del monte gallego, al incluir las formaciones forestales con menores unidades mínimas cartografiables que otras fuentes disponibles y no obtener la información únicamente a partir de los porcentajes de ocupación indicados en la base de datos del SIOSE05. El SIOSE07-08 es capaz de proporcionar información de detalle de la cobertura forestal teniendo en cuenta el complejo paisaje del territorio de Galicia que, en su mayor parte, está ocupado con coberturas mixtas. En el caso de Galicia, la elevada fragmentación y discontinuidad de los usos, obligó a tener en cuenta multitud de teselas, aunque muchas fueran de tamaño reducido, para una correcta delimitación de la superficie forestal. Nuestros resultados comparan la información sobre superficie forestal con el fin de evaluar la armonización y fiabilidad en la información espacial sobre la superficie forestal.

Con relación a otras fuentes cartográficas estudiadas, se observan importantes diferencias con el dato del CLC06 y USO_25 en la cuantificación del área forestal total, con diferencias de 475.000 ha y 562.000 ha menos, respectivamente. Estas fuentes además subestiman también la superficie forestal con relación al SIOSE07-08 en su evaluación a nivel provincial y comarcal, pudiendo alcanzar diferencias en torno al $50 \%$ en determinadas comarcas. Estas fuentes carecen de muchas de las ventajas indicadas para el SIOSE07-08 debido, entre otros factores, a la escala de digitalización, a las fuentes de información empleadas y a las unidades mínimas cartografiables. Estas características condicionan enormemente la precisión, forzando que algunas coberturas del suelo se clasifiquen en clases que no les corresponden lo que repercute en los resultados obtenidos, tal y como se ha ilustrado en este trabajo. 
En concreto, en relación al CLC06 las diferencias pueden ser debidas a errores de generalización derivada de la escala de trabajo, 1: 100.000 frente a 1: 25.000 del SIOSE, y/o a posibles errores de clasificación de las coberturas del suelo. Errores del CLC que ya aparecen documentados en otros trabajos (Felicísimo y Sánchez, 2002).

Por otro lado, la similitud que hemos hallado en la cuantificación de la superficie forestal entre el IFN4 y el SIOSE07-08 tanto a nivel regional como a nivel de distribución espacial, provincial y comarcal, es un indicador clave de la validez de la actualización propuesta, SIOSE07-08. Dado que el Inventario Forestal Nacional es el proyecto que proporciona información de detalle a nivel estatal sobre los bosques y su evolución, tanto desde un punto de vista dasonómico como ecológico, el IFN4 se constituye en una fuente de referencia básica y completa a utilizar para el análisis de los bosques. Con este trabajo concluimos que la actualización y depuración de la información del SIOSE05, SIOSE07-08, presenta una alta fiabilidad, obteniendo datos muy similares al IFN4. Este resultado, ofrece una garantía para potenciales actualizaciones que de forma similar se realicen del SIOSE05 para la cuantificación y localización de otras coberturas (agrícola, matorral, superficie arbolada, urbana) en el territorio.

Finalmente, comentar que este trabajo demuestra como la diversidad de coberturas y metodologías dificulta la comparación entre fuentes y la posibilidad de establecer un dato preciso de superficie forestal. A este panorama cartográfico se ha incorporado una nueva fuente de información que habrá que considerar a corto plazo, el dato LiDAR (Light Detection And Ranging), ya que aportará una delimitación más exacta y detallada de las formaciones forestales. El LiDAR es un sistema de teledetección activa, basado en pulsos de luz laser del que se puede derivar información forestal (identificación de formaciones y sus características). Además, ofrece la posibilidad de medir la altura de los elementos, por lo que es posible identificar entre vegetación arbolada y arbustiva, algo sin duda, con importantes implicaciones en la comunidad autónoma que hemos tomado como estudio de caso en este documento, por su ya comentada incidencia en el sector forestal español. En la actualidad, se dispone del vuelo del PNOA (http://www.ign.es/PNOA/caracteristicas_tecnicas.html) en este formato con una resolución de punto de $2 \mathrm{~m}$ y una precisión en la coordenada $\mathrm{Z}$ de 0,2 $\mathrm{m}$. Esta nueva tecnología permitirá disponer de datos más fiables sobre la superficie forestal, facilitando la valoración del grado de calidad de las restantes fuentes cartográficas tradicionales analizadas aquí. 